\title{
Comparative performance assessment of beam hardening correction algorithms applied on simulated data sets
}

\author{
Wenchao $\mathrm{Cao}^{1}$, Tao $\mathrm{Sun}^{2}$, Greet Kerckhofs ${ }^{3}$, Gemma \\ Fardell $^{4}$, Ben Price ${ }^{4}$, Wim Dewulf ${ }^{1}$ \\ Department of Mechanical Engineering, KU Leuven, Celestijnenlaan 300, 3001, \\ Leuven, Belgium ${ }^{1}$ \\ Department of Nuclear Medicine \& Molecular Imaging, KU Leuven, UZ \\ Herestraat 49, 3000, Leuven, Belgium ${ }^{2}$ \\ Biomechanics lab, Institute of Mechanics, Materials, and Civil Engineering, \\ Université Catholique de Louvain, Louvain-la-Neuve, Belgium ${ }^{3}$ \\ X-Tek Systems Ltd (Nikon Metrology UK Ltd), Unit 5, Icknield Industrial \\ Estate, Tring, UK ${ }^{4}$ \\ E-mail: wenchao.cao@kuleuven.be
}


Abstract. Beam hardening artefacts deteriorate the reconstructed image quality in industrial computed tomography. The appearances of beam hardening artefacts can be cupping effects or streaks. They impair the image fidelity to the object being scanned. This work aims at comparing a variety of commonly used beam hardening correction algorithms in the context of industrial computed tomography metrology. We choose four beam hardening correction algorithms of different types for the comparison. They are a single-material linearization algorithm, a multimaterial linearization algorithm, a dual-energy algorithm and an iterative reconstruction algorithm. Each beam hardening correction algorithm is applied to simulated data sets of a dual-material phantom consisting of multiple rods. The comparison is performed on data sets simulated both under ideal conditions and with the addition of quantum noise. The performance of each algorithm is assessed with respect to its effect on the final image quality (contrast-to-noise ratio, spatial resolution), artefact reduction (streaks, cupping effects) and dimensional measurement deviations. The metrics have been carefully designed in order to achieve a robust and quantifiable assessment. The results suggest that the single-material linearization algorithm can reduce beam hardening artefacts in the vicinity of one material. The multimaterial linearization algorithm can further reduce beam hardening artefacts induced by the other material and improve the dimensional measurement accuracy. The dual-energy method can eliminate beam hardening artefacts, and improve the low contrast visibility and dimensional measurement accuracy. The iterative algorithm is able to eliminate beam hardening streaks. However, it induces aliasing patterns around the object edge, and its performance depends critically upon computational power. The contrast-to-noise ratio and spatial resolution are decreased by noise. Noise also increases the difficulty of image segmentation and quantitative analysis.

Keywords: Beam hardening, performance assessment, dimensional metrology, computed tomography 


\section{Introduction}

Beam hardening is a major source of image artefacts in X-ray CT (Herman 2009). In the conventional filtered back-projection (FBP) reconstruction algorithm, the $\mathrm{X}$-ray spectrum is assumed to be monochromatic. The relationship between the incident beam intensity $I_{0}$ and the transmitted X-ray beam intensity $I$ after passing through a single material, is modelled as,

$$
-\ln \left(\frac{I}{I_{0}}\right)=P_{m}=\int_{0}^{l} \mu(x) d x
$$

where $P_{m}$ denotes the logarithmic monochromatic projection value, $\mu(x)$ is the attenuation coefficient at position $\mathrm{x}$ along the ray, which characterizes how easily it can be penetrated by X-ray. It is energy independent in the assumption of FBP.

In the lab-based CT systems, the X-ray tube emits polychromatic X-rays, so the logarithmic transmission is as follows,

$$
\begin{aligned}
\ln \left(\frac{I_{0}}{I}\right) & =P_{p} \\
& =\ln \left(\frac{\int S(E) d E}{\int S(E) \exp \left(-\int_{0}^{l} \mu(x, E) d x\right) d E}\right)
\end{aligned}
$$

where $P_{p}$ denotes the logarithmic polychromatic projection value, $\mu(x, E)$ denotes the attenuation coefficient at position $\mathrm{x}$ under energy $E$. It is energy dependent. Ignoring the polychromatic nature of $S(E)$ in FBP algorithms produces inconsistent values in the reconstructed tomogram in the form of beam hardening artefacts.

Linearization (Section 2.1) is the simplest BHC algorithm, which uses a lookup table to 'linearize' the polychromatic image intensities (Brooks \& Di Chiro 1976, McDavid et al. 1977, Herman \& Trivedi 1983, Hammersberg \& Mångård 1998). Since the look-up table is material dependent, the method fails with multimaterial objects. Linearization can be combined with post-processing strategies (e.g. segmentation), for multi-material correction (Joseph \& Spital 1978, Nalcioglu \& Lou 1979, Joseph \& Ruth 1997) (Section 2.2). However, beam hardening causes difficulty in the segmentation of a low attenuating material. Creating the actual linearization curves that agree well with all segments is another challenge since the energy spectrum of a specific X-ray system is usually unavailable. Dualenergy techniques (Section 2.3) decompose the material attenuation coefficient 
into a linear combination of two basis functions (Alvarez \& Macovski 1976, Lehmann et al. 1981, Kalender et al. 1986, Liu et al. 2009). The weights of the two basis functions can be solved by obtaining scanned data sets at two different X-ray spectra. Since the energy-dependent information is stored in the basis functions, a pseudo monochromatic image can be produced. The need for a specific hardware configuration limits the application of the dual-energy technique. Iterative algorithms (Section 2.4) can reduce beam hardening artefacts by implementing the energy-dependent information in the reconstruction model (Yan et al. 2000, De Man et al. 2001, Elbakri \& Fessler 2002, Brabant et al. 2012, Van Slambrouck \& Nuyts 2012). Nonetheless, it usually can only achieve a compromise between the computational capacity and algorithm performance.

Although these algorithms have been widely investigated in medical CT, there is a lack of research on their effectiveness in industrial CT. The main cause of beam hardening in medical $\mathrm{CT}$ is bone and surgical implants. Since industrial parts, which are made of multi-material components such as plastic and high density (e.g. copper \& steel) metal inserts, are generally more complex and Xray absorbing, a stronger appearance of artefacts compared to the medical data is likely to be observed. Therefore, the knowledge of how the BHC algorithms perform on industrial samples is of great importance.

This study presents an in-depth BHC algorithm comparison for industrial CT. Since this study takes various factors of the algorithm performance into account, it provides insights into the advantages and disadvantages of each algorithm and assists the choice of algorithm to meet specific industry needs. The study also highlights the remaining challenges in industrial BHC. The methods investigated in this study are single-material linearization (Herman 1979), multi-material linearization (Joseph \& Spital 1978), dual-energy (Alvarez \& Macovski 1976), and IMPACT iterative method (De Man et al. 2001). We compare these methods with respect to their performance on image quality, the removal of image artefacts, and the influence on dimensional accuracy.

This study is based on simulation for two reasons. In real CT scanning, there are several mechanisms which produce artefacts with similar appearances. By using simulation we can exclude these mechanisms and focus on studying the effect of beam hardening. In addition, monochromatic images are the 'ground truth' for beam hardening. They can only be acquired in simulation. 


\section{Theory}

\subsection{Single-material linearization}

The single-material linearization method used in our comparison is proposed by (Herman \& Trivedi 1983). For single material, the non-linear relationship between the polychromatic projection image intensity $\mathrm{P}_{p}$ and its corresponding monochromatic value $P_{m}$ can be approximated by a series of polynomials,

$$
P_{m}=f\left(P_{p}\right)=a_{n} P_{p}^{n}+a_{n-1} P_{p}^{n-1}+\ldots+a_{0}
$$

where $n$ is the order of the polynomial, $a_{0}, a_{1}, a_{2}, \ldots, a_{n}$ are the coefficients. Typically, polynomial order $n$ between 1 and 4 is used. For high-density materials, higher polynomial degrees may be needed.

\subsection{Multi-material linearization}

Multi-material linearization method was implemented based on JS method (Joseph \& Spital 1978). This section assumes an object that is comprised of two materials. In the conventional FBP reconstruction algorithm, the X-ray spectrum is assumed to be monochromatic. The relationship between the transmitted X-ray beam intensity $I$ after passing through a single material and the incident beam intensity $I_{0}$ is therefore modelled as

$$
-\ln \left(\frac{I}{I_{0}}\right)=p_{m}=\int \mu_{H} d l_{H}+\int \mu_{S} d l_{S}
$$

where $p_{m}$ stands for the logarithmic monochromatic projection value, $\mu_{H}$ and $\mu_{S}$ are the attenuation coefficients of the hard and soft material, respectively; $l_{H}$ and $l_{S}$ are their corresponding propagation lengths. The attenuation coefficient is a function of the propagation length.

In practice, the logarithmic transmission is modelled as:

$$
\begin{aligned}
p_{p} & =-\ln \left(\frac{\int S(E) \exp \left(-\int_{0}^{l_{H}} \mu_{1}(x, E) d x-\int_{0}^{l_{S}} \mu_{2}(x, E) d x\right) d E}{\int S(E) d E}\right) \\
& =-\ln \left(\frac{\int S(E) \exp \left(-\int_{0}^{l_{H}} \mu_{1}(x, E) d x\right) d E}{\int S(E) d E}\right)-\ln \left(\frac{\int S^{\prime}(E) \exp \left(-\int_{0}^{l_{S}} \mu_{2}(x, E) d x\right) d E}{\int S^{\prime}(E) d E}\right) \\
& =p_{H}+p_{S}
\end{aligned}
$$


where $S^{\prime}(E)=S(E) \exp \left(-\int_{0}^{l_{1}} \mu_{1}(x, E) d x\right)$;

In the above equations, $p_{p}$ is the logarithmic polychromatic projection value; $p_{H}$ and $p_{S}$ are the polychromatic contributions of the hard and soft materials.

As seen in Equation 5, despite the material distribution along an X-ray beam, $p_{p}$ only changes with the propagation length. The materials can be distributed in any order without changing the signal on the detector (Nalcioglu \& Lou 1979). Therefore, we assume that the X-ray beam enters into a slab of the hard material and then enters into the soft material, which means the former can be seen as a filter of the latter. $S^{\prime}(E)$ is the 'hardened' spectrum by the hard material with propagation length $l_{H}$. Beam hardening artefacts result from simplifying the spectrum $S(E)$ into a single energy level in the FBP algorithm.

There are two stages of the JS method. The first stage involves building a LUT for soft material correction. In Equation $5, S^{\prime}(E)$ is a function of $l_{H}$. After discretizing the propagation length with a certain step, for each step a polynomial fit $f$ is used to linearize the polychromatic contribution of the soft material $\left(p_{S}\right)$ to the monochromatic contribution $\left(m_{S}\right)$ under the corresponding $S^{\prime}(E)$ (Equation 6).

$$
m_{S}=f\left(p_{s}\right)=a_{n} \times p_{s}^{n}+a_{n-1} \times p_{s}^{n-1}+\ldots+a_{0}
$$

For each discrete propagation length $l_{H}$ through the hard material, the LUT contains $4^{\text {th }}$ order polynomial coefficient for the soft material linearization. Because interpolation is needed to index values from the LUT, a small enough incremental step of $l_{H}$ is required to minimise the interpolation error. (Hammersberg \& Mångård 1998) suggested using a step size of $L_{H} / 1000$, where $L_{H}$ is the largest propagation length of the hard material.

In the second stage, after initial reconstruction, the hard material is segmented. The propagation length through the hard material corresponding to each pixel is traced. The monochromatic contribution of the hard material is calculated directly with:

$$
m_{H}=\mu_{H}\left(E_{0}\right) \times l_{H}
$$

$E_{0}$ is the monochromatic energy that the image is tuned at. The monochromatic contribution of the soft material is linearized by the polynomial coefficients in the LUT. 


\subsection{Dual-energy}

The dual-energy method used in the comparison is in accordance with (Alvarez \& Macovski 1976). It formulates the attenuation coefficient as follows,

$$
\mu(E)=a_{1} f_{P E}+a_{2} f_{K N}
$$

where

$$
\begin{gathered}
f_{P E}=\frac{1}{E^{3}} \\
f_{K N}(\alpha)=\frac{1+\alpha}{\alpha^{2}}\left[\frac{2(1+\alpha)}{1+2 \alpha}-\frac{1}{\alpha} \ln (1+2 \alpha)\right]+\frac{1}{2 \alpha} \ln (1+2 \alpha)
\end{gathered}
$$

$f_{P E}$ is the approximated energy dependence of photoelectric effect, and $f_{K N}$ gives the energy dependence of Compton scattering. The reconstruction of $a_{1}$ and $a_{2}$ requires the measurement of line integrals $A_{1}=\int a_{1} d s$ and $A_{2}=\int a_{2} d s$ at every pixel in the projections of the object. To obtain $A_{1}$ and $A_{2}$, two independent pieces of image intensity information must be available, which can be done by making measurements at two different energies:

$$
\begin{aligned}
I_{h} & =\int S_{h}(E) \exp \left[-A_{1} f_{P C}(E)-A_{2} f_{K N}(E)\right] d E \\
I_{l} & =\int S_{l}(E) \exp \left[-A_{1} f_{P C}(E)-A_{2} f_{K N}(E)\right] d E
\end{aligned}
$$

$S_{h}$ and $S_{l}$ represent respectively the X-ray spectra of the high and low energy measurements. If the spectra are known, $A_{1}$ and $A_{2}$ can be solved with iterative methods. We can subsequently generate pseudo-monochromatic projections with $A_{1}$ and $A_{2}$.

\subsection{Iterative reconstruction method}

The iterative reconstruction method IMPACT (iterative maximum likelihood polychromatic algorithm for CT) we used in the comparison was proposed by (De Man et al. 2001).

As implied by its name, IMPACT finds the solution by maximising the loglikelihood function. In the context of CT, it is a Poisson likelihood,

$$
L=\Sigma_{i=1}^{I}\left(y_{i} \ln \hat{y}_{i}-\hat{y}_{i}\right)
$$

where $i$ is the pixel index, $\hat{y}_{i}$ is the estimated image intensity and $y_{i}$ the measured one. 
The polychromatic projection formula is given by,

$$
\hat{y}_{i}=\Sigma_{k} \hat{y}_{i k}=\Sigma_{k} b_{i k} \exp \left(-\Theta_{k} \Sigma_{j} l_{i j} \theta\left(\mu_{j, r e f}\right)-\Phi_{k} \Sigma_{j} l_{i j} \phi\left(\mu_{j, r e f}\right)\right)
$$

where $l_{i j}$ denotes the intersection length of projection line i and pixel $\mathrm{j} ; \mu_{j}$ denotes the linear attenuation coefficient in voxel $\mathrm{j} ; \mu_{j, r e f}$ is the reconstructed linear attenuation coefficient in pixel $\mathrm{j}$ at the reference energy. The attenuation coefficient is decomposed using the Alvarez dual-energy formula. $\Theta_{k}$ and $\Phi_{k}$ are the energy dependence of respectively Compton scattering and photo-electric effect, and $\theta$ and $\phi$ are the material dependence. We assume that $\theta$ and $\phi$ are unambiguously determined by the attenuation at a chosen reference energy: $\theta_{j}=\theta\left(\mu_{j, r e f}\right)$ and $\phi_{j}=\phi\left(\mu_{j, r e f}\right)$. We assume that each voxel can be modelled as a mixture of two base substances. The algorithm for updating the attenuation coefficient would be:

$$
\Delta \mu_{j}=\frac{\phi_{j}^{\prime} \Sigma_{i} l_{i j} e_{i} Y_{i}^{\Phi}+\theta_{j}^{\prime} \Sigma_{i} l_{i j} e_{i} Y_{i}^{\Theta}}{\phi_{j}^{\prime} \Sigma_{i} l_{i j} M_{i}+\theta_{j}^{\prime} \Sigma_{i} l_{i j} N_{i}}
$$

where

$$
\begin{array}{cc}
\phi_{j}^{\prime}=\frac{\partial \phi}{\partial \mu_{j}} & \theta_{j}^{\prime}=\frac{\partial \theta}{\partial \mu_{j}} \\
e_{i}=1-\frac{y_{i}}{\hat{y}_{i}} & Y_{i}^{\Theta}=\Sigma_{k} \Theta_{k} \hat{y}_{i k} \\
Y_{i}^{\Phi}=\Sigma_{k} \Phi_{k} \hat{y}_{i k} & Y_{i}^{\Theta \Theta}=\Sigma_{k} \Theta_{k}^{2} \hat{y}_{i k} \\
Y_{i}^{\Phi \Phi}=\Sigma_{k} \Phi_{k}^{2} \hat{y}_{i k} & \\
Y_{i}^{\Phi \Theta}=\Sigma_{k} \Phi_{k} \Theta_{k} \hat{y}_{i k} & \\
M_{i}=\left(\Sigma_{h} l_{i h} \phi_{h}^{\prime}\right)\left(Y_{i}^{\Phi \Phi} e_{i}+\frac{y_{i}\left(Y_{i}^{\Phi}\right)^{2}}{\hat{y}_{i}^{2}}\right)+\left(\Sigma_{h} l_{i h} \theta_{h}^{\prime}\right)\left(Y_{i}^{\Phi \Theta} e_{i}+\frac{y_{i} Y_{i}^{\Phi} Y_{i}^{\Theta}}{\hat{y}_{i}^{2}}\right) \\
N_{i}=\left(\Sigma_{h} l_{i h} \phi_{h}^{\prime}\right)\left(Y_{i}^{\Phi \Theta} e_{i}+\frac{y_{i} Y_{i}^{\Phi} Y_{i}^{\Theta}}{\hat{y}_{i}^{2}}\right)+\left(\Sigma_{h} l_{i h} \theta_{h}^{\prime}\right)\left(Y_{i}^{\Theta \Theta} e_{i}+\frac{y_{i}\left(Y_{i}^{\Theta}\right)^{2}}{\hat{y}_{i}^{2}}\right)
\end{array}
$$

Each update requires eight forward- and back- projections.

\section{Materials and methods}

\subsection{Virtual phantom design}

A multi-material virtual phantom is designed for the simulation framework of this study. The phantom is comprised of three metal rods, two solid plastic rods, 
and one hollow plastic rod. The metal material is titanium (Ti), and the plastic material is polyphenylene sulphide (PPS). The phantom features multiple external and internal dimensions (Figure 1). Details of the phantom geometry are given in Table 1.

\begin{tabular}{|l|l|}
\hline Phantom element & Nominal radius (mm) \\
\hline Metal1 & 2.0 \\
\hline Metal2 & 1.5 \\
\hline Metal3 & 1.5 \\
\hline PPS1 & 1.5 \\
\hline PPS2 & 1.5 \\
\hline PPS3 - external & 2.5 \\
\hline PPS3 - internal & 1.5 \\
\hline
\end{tabular}

Table 1: Phantom element nominal dimensions.

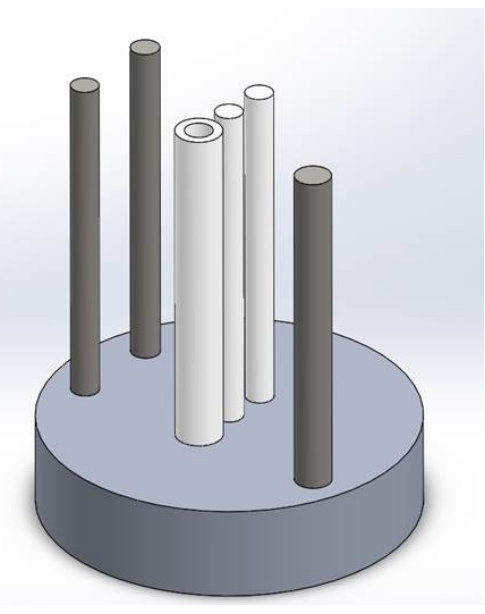

(a)

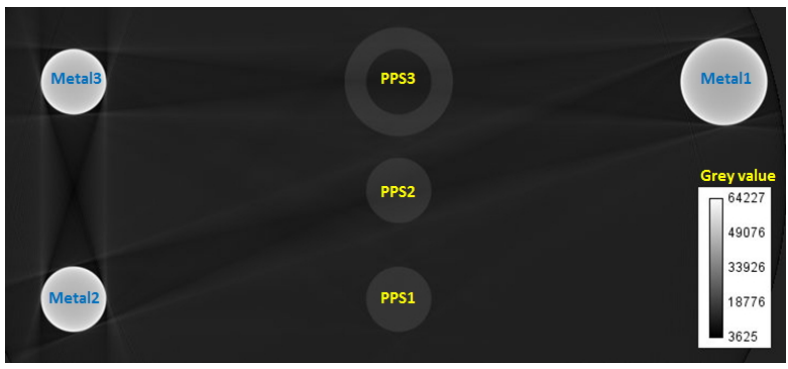

(b)

Figure 1: Virtual phantom. (a) A 3D drawing of the phantom. (b) Reconstructed central slice of the simulated phantom. 


\subsection{Contrast-to-noise ratio (CNR)}

The CNR investigates to what extent the BHC algorithms degrade contrast resolution of the PPS rods or increase the noise. The following formula is used:

$$
C N R=\frac{\left|M_{m}-M_{b}\right|}{C \times\left(1+\sqrt{\sigma_{m}^{2}+\sigma_{b}^{2}}\right)}
$$

where $M_{m}$ and $\sigma_{m}$ are the mean and standard deviation of the ROI image intensity within one of the PPS rods (either $\mathrm{ROI}_{1}$ or $\mathrm{ROI}_{2}$ ), $M_{b}$ and $\sigma_{b}$ are the mean and standard deviation of the background image intensity (within $\mathrm{ROI}_{b}$, see Figure 2), and $\mathrm{C}$ is the maximum grey value of a certain type of images. In a 16-bit unsigned image, $\mathrm{C}$ equals $65535 . \mathrm{ROI}_{1}$ and $\mathrm{ROI}_{2}$ have a radius equal to $1 / 3$ radius of the rod. $\mathrm{ROI}_{b}$ is within the bottom right rectangle of $50 \times 25$ pixels. This formula is adjusted from the original definition of CNR (Bushberg \& Boone 2011). By including $\sigma_{m}$ in the denominator, we take the influence of beam hardening on the image uniformity into account. The scalar 1 in the denominator prevents CNR calculation tending to infinity in noise-free simulations. The upper and lower bounds of $\mathrm{CNR}_{\text {max }}$ and $\mathrm{CNR}_{\text {min }}$ are therefore 1 and 0 . The $\mathrm{CNR}$ values of $\mathrm{ROI}_{1}$ and $\mathrm{ROI}_{2}$ reveal two different kinds of information. $\mathrm{ROI}_{1}$ lies in a beam hardening free area; hence its CNR indicates the highest visibility PPS can achieve. $\mathrm{ROI}_{2}$ lies in an area where the low contrast visibility is impaired by the beam hardening streaks. If a $\mathrm{BHC}$ algorithm brings the $\mathrm{CNR}$ of $\mathrm{ROI}_{2}$ to a value close to that of $\mathrm{ROI}_{1}$, it is an indication of image quality improvement.

As the background is hardly affected by an algorithm, the change of the CNR value is mainly caused by $M_{m}, \sigma_{m}$, and $\sigma_{b}$. They correspond to the material and noise information, respectively. Figure 3 illustrates various CNR values for both noise-free and noisy data sets. Comparing the two columns of the figure, we can find that the CNR reduction caused by image intensity decrease or noise appears to be different. In the left column, the rod gradually gets darker and less visible. In the right column, the image intensity of the rod does not change, but it gets less legible with increasing noise.

An experiment is conducted to assess the sensitivity of the modified CNR metric, i.e. to estimate the order of magnitude of the CNR metric variation that might be expected despite similar imaging conditions. Scans of two single rods of respectively PPS plastic and Ti are simulated for this purpose. Figure 4a depicts the regions of interest (ROIs) used in the calculation. The material ROI is a concentric red circle with a $1 / 3$ radius of the material rod. A blue square is at 


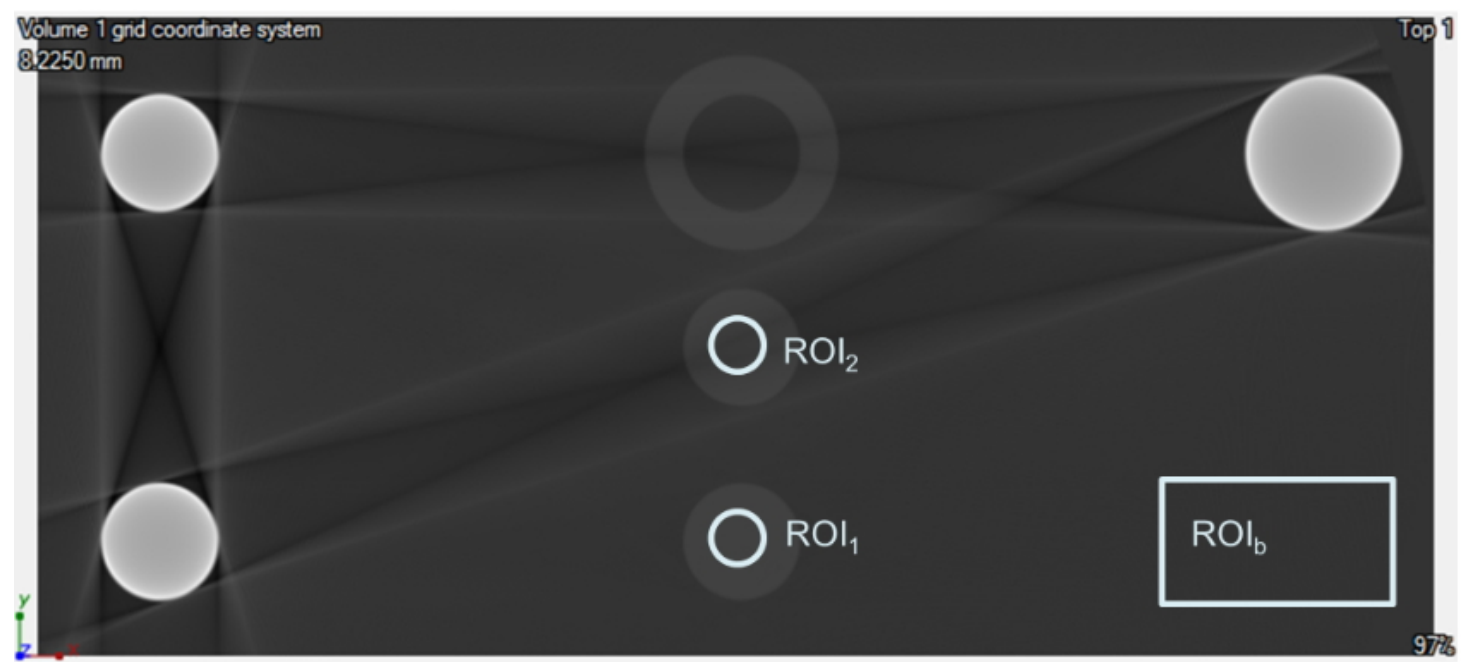

Figure 2: ROI definition of CNR. The central slice of the reconstructed stack is used for CNR data capturing.

$0.1 \mathrm{~mm}$ inside the image edge and a blue circle at $0.1 \mathrm{~mm}$ outside the material edge. The background ROI comprises the pixels in-between. The variation of the CNR value is accessed by calculating 32 additional CNR values for 32 different background ROIs (Figure 4b). The background ROIs have size $10 \times 10$ pixels and are centred on a circle with a radius that equals $1.5 \times$ phantom radius. Multiple CNR values are calculated with the background ROIs. The standard deviation of the calculated CNRs is defined as twice of the variation range of CNR, which is plotted as error bars on the figures.

The results are shown in Figure 5. Contrast resolution is lowest in the polychromatic data set and highest in the monochromatic data set as can be expected. Moreover, a smaller CNR is found with the noisy data set as compared to the noise free one, because $\sigma_{m}$ and $\sigma_{b}$ are larger. CNR of the PPS data set is only slightly smaller than for the Ti data set, which is due to the fact that the PPS phantom was simulated at lower energy. 
(a)

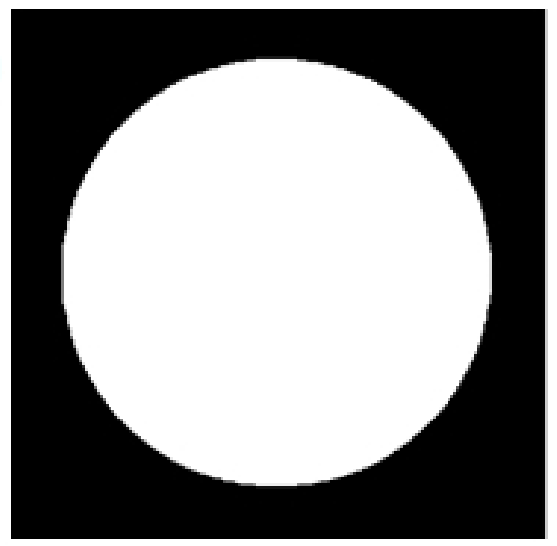

$\mathrm{CNR}=1$

(b)

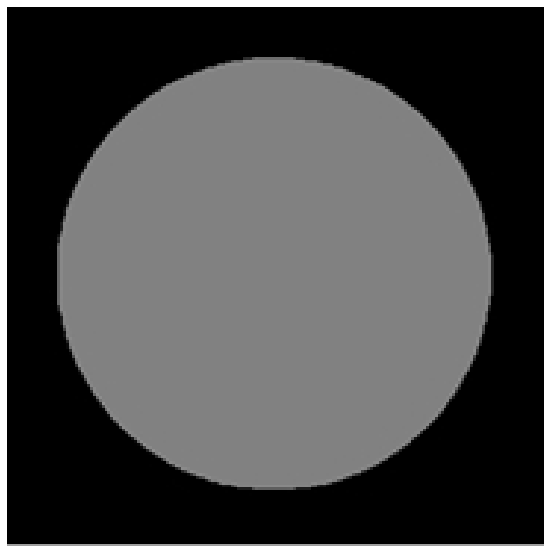

$\mathrm{CNR}=0.5$

(C)

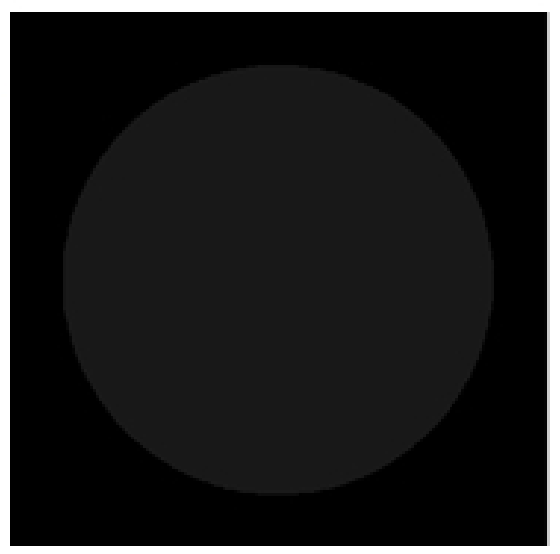

$\mathrm{CNR}=0.01$ (d)

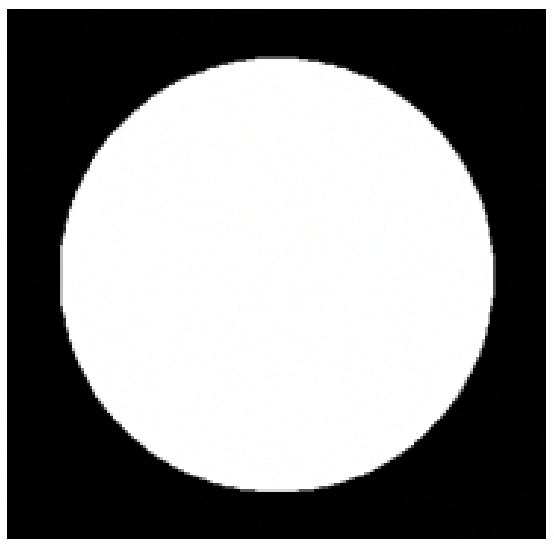

$\mathrm{CNR}=0.9$

(e)

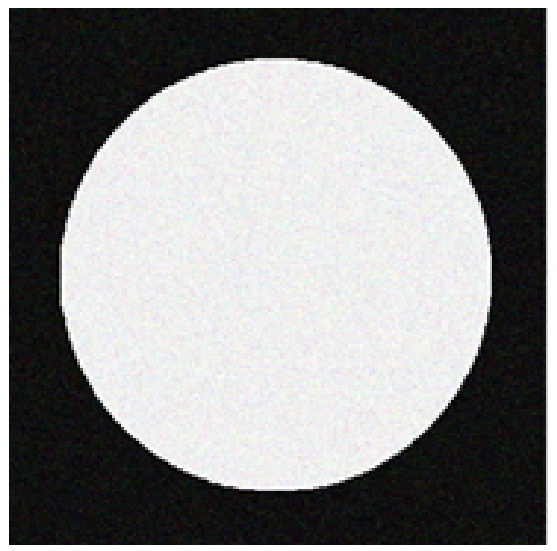

CNR $=0.5$

(f)

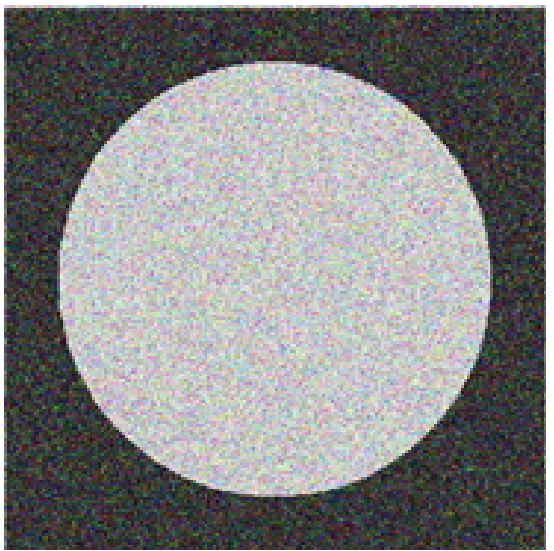

$\mathrm{CNR}=0.01$

Figure 3: An illustration of different CNR values. The left column shows images with no noise, and the right shows those with noise. 


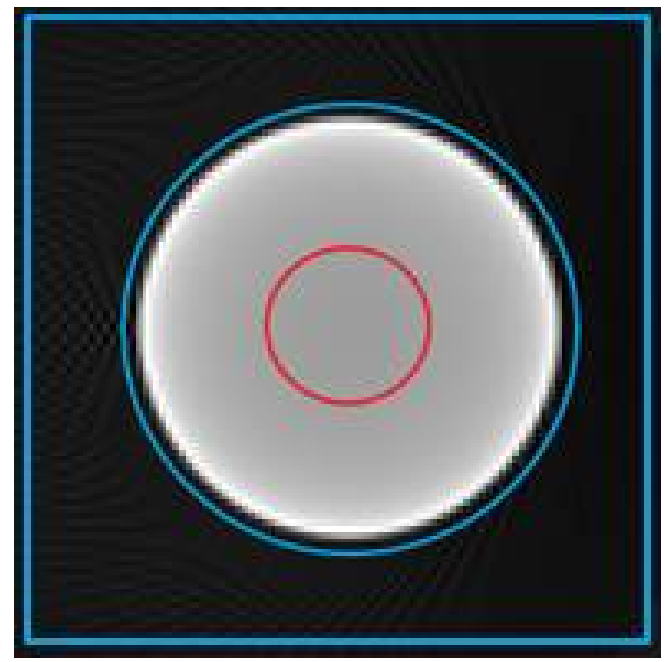

(a)

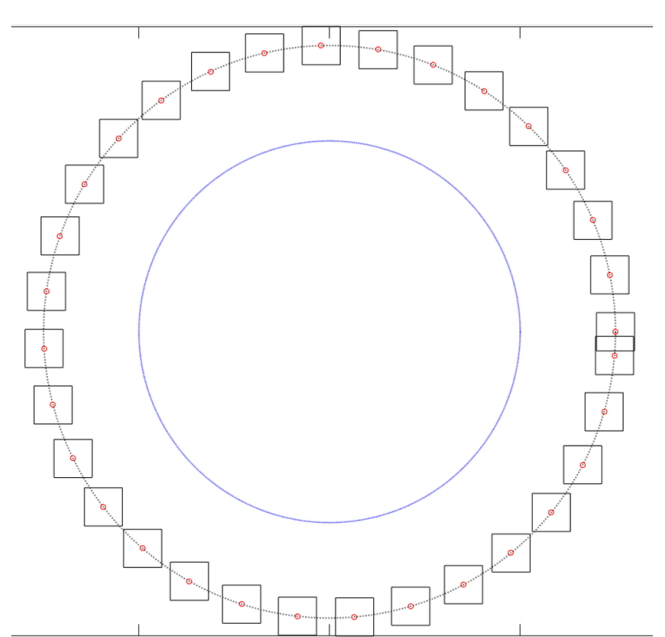

(b)

Figure 4: (a) ROI definition for CNR. (b) A schematic diagram of the background ROIs selection for CNR uncertainty calculation.

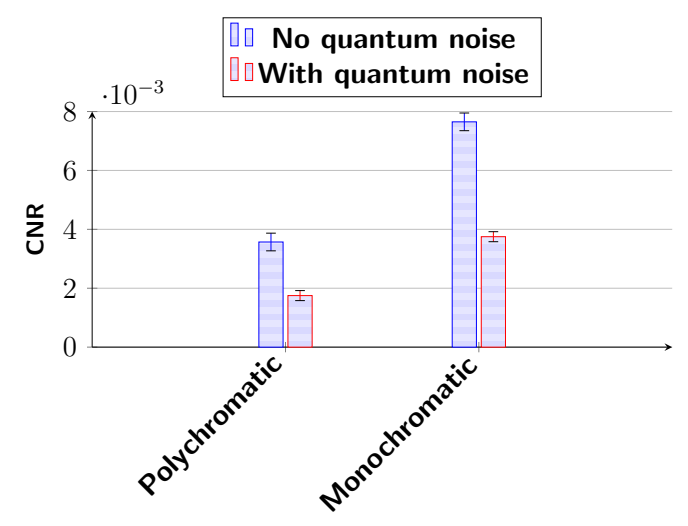

(a) Single Ti rod.

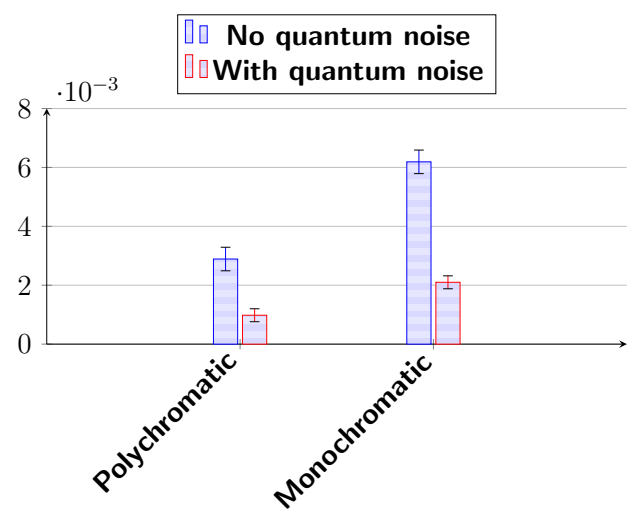

(b) Single PPS rod.

Figure 5: CNR results on mono-material rods. 


\subsection{Modulation transfer function (MTF)}

This metric aims at investigating whether the BHC algorithms affect the spatial resolution of the reconstructed image. Cupping effects result in a more discernible feature because it introduces raised intensity values at the edge. By comparing the spatial resolutions without and with beam hardening, researchers have been able to prove that the presence of beam hardening enhances the spatial resolution of an image (Van de Casteele et al. 2004). Whereas the study of (Van de Casteele et al. 2004) examined how the artefacts affected the local spatial resolution, our study aims at monitoring the influences of the algorithms on the image spatial properties.

A resolution measure which is often used by manufacturers is the $10 \% \mathrm{MTF}$ value (Friedman et al. 2013). MTF calculation procedure was adapted from (Friedman et al. 2013). In (Friedman et al. 2013), the reconstructed slices were averaged together along the $z$ direction on a voxel-by-voxel basis to create a single $2 \mathrm{D}$ axial image. Instead, only the central slice was selected for a $2 \mathrm{D} \mathrm{MTF}$ calculation in our research. The steps of our method can be seen in Figure 6, and are briefly explained below:

(i) Three ROIs need to be defined (Figure 6). The calculation ROI is denoted by $\mathrm{ROI}_{c}$. It is inside the red square, which is $75 \times 75$ pixels in dimension. The background ROI is denoted by $\mathrm{ROI}_{b}$. It is the region between the red and blue squares. The blue square is $0.1 \mathrm{~mm}$ inside the image. The material ROI is denoted by $\mathrm{ROI}_{m}$. It is inside the red circle, which is a circle with radius equals to $1 / 3$ radius of the rod.

(ii) The grey values inside $\mathrm{ROI}_{c}$ are normalised using the following formula:

$$
x_{i, j}^{n o r m}=\frac{x_{i, j}-M_{b}}{M_{m}-M_{b}}
$$

where $x_{i, j}$ represents the grey value at the pixel $(i, j)$ in $\mathrm{ROI}_{c}, \mathrm{M}_{m}$ and $\mathrm{M}_{b}$ are the average grey values of respectively $\mathrm{ROI}_{m}$ and $\mathrm{ROI}_{b}$.

(iii) The centre $\mathrm{O}$ of the rod is determined using VG by fitting a circle.

(iv) The oversampled edge-spread function (ESF) is calculated by mapping all points in $\mathrm{ROI}_{c}$ to their polar-coordinate equivalent and re-binning based on distance from $\mathrm{O}$ with a sub-pixel bin size of $0.01 \mathrm{~mm}$ in width.

(v) The ESF is differentiated to produce the corresponding line-spread function (LSF). 
(vi) The MTF is then calculated by taking the Fourier transform of the LSF, and the $10 \%$ MTF can be read off.

To exclude the non-separable spatial property change caused by beam hardening, we need to define our ROI for MTF calculation beam hardening free. The ROIs are shown in Figure $7 . \mathrm{ROI}_{c}$ and $\mathrm{ROI}_{m}$ are for the MTF calculation. The former is within the square concentric to the rod, which size is $80 \times 80$ pixels, and $\mathrm{ROI}_{m}$ is centred on the rod with a $1 / 3$ radius of the rod. $\mathrm{ROI}_{b}$ is the right bottom rectangle, which size is $50 \times 25$ pixels.

Although the the $10 \% \mathrm{MTF}$ value reveals the spatial resolution of an image, the main purpose of this study is to monitor if there is any change in the image caused by a BHC algorithm. An experiment is conducted to assess the sensitivity of the MTF metric, i.e. to estimate the order of magnitude of the MTF indicator variation that might be expected despite similar imaging conditions. A single rod is repetitively simulated five times at different positions (labeled on Figure 8) in the volume. The $10 \%$ MTF values are calculated for each simulation. This set yields a mean value and a standard deviation of 11.4 and 0.27 respectively. Therefore, fluctuations lower than $5 \%$ can be considered insignificant. 
Step 1-3: preparations
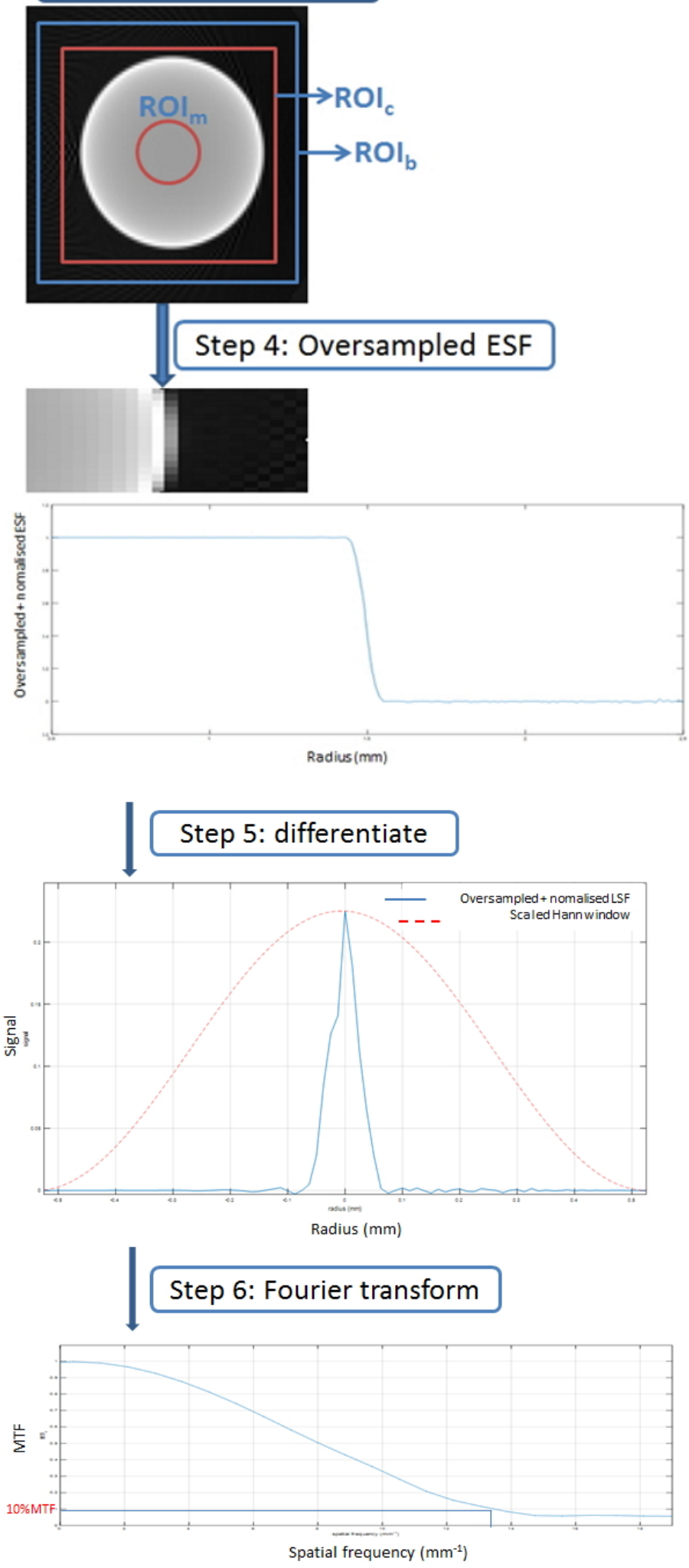

Figure 6: Flowchart of the MTF calculation procedure. 


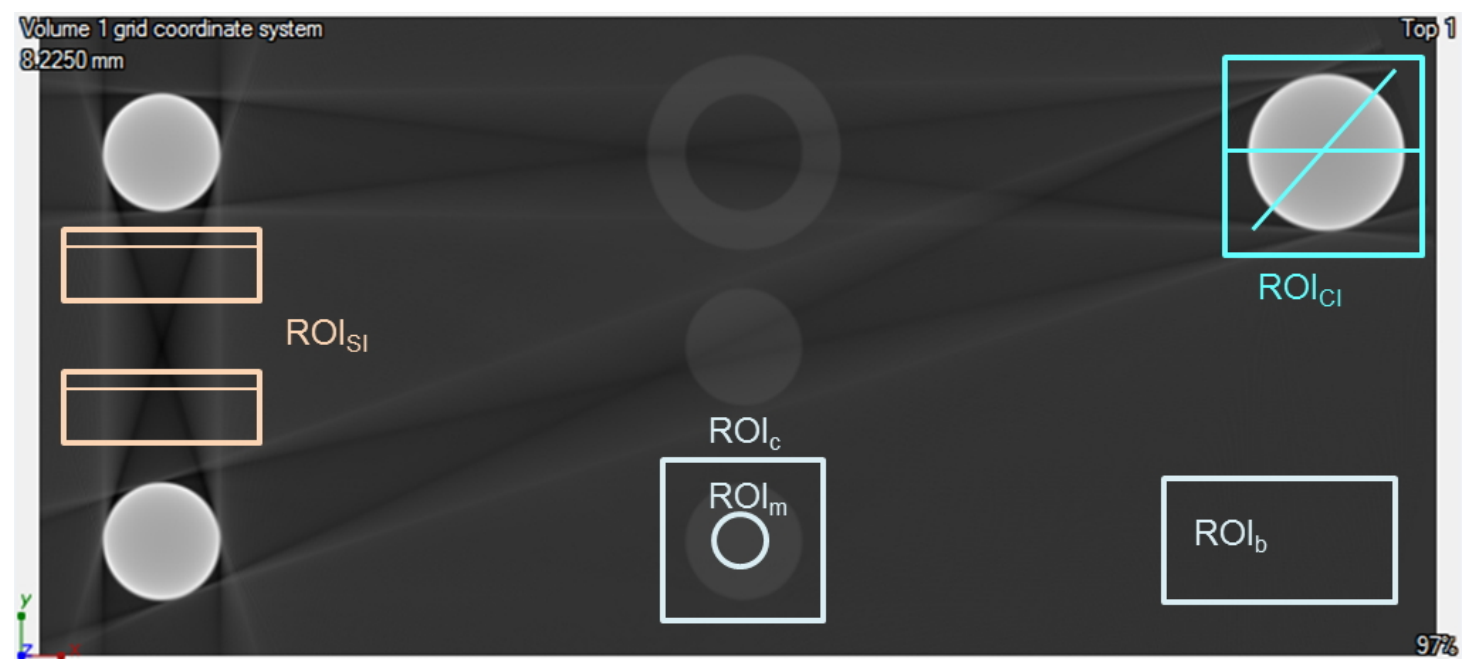

Figure 7: ROI definitions of multiple metrics. Within each $\mathrm{ROI}_{S I}$ and $\mathrm{ROI}_{C I}$, several line profiles are drawn. For reason of clarity, only two are shown in each ROI.
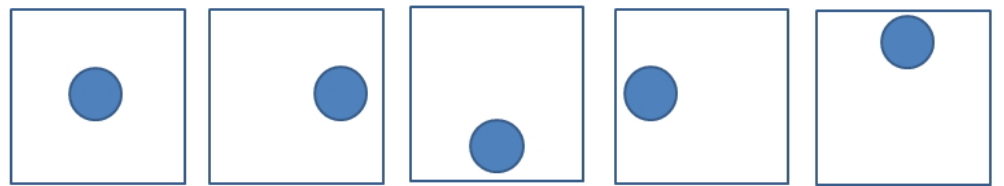

Figure 8: A schematic of the five positions. 


\subsection{Streak index (SI)}

The SI metric has been developed exclusively for this BHC algorithm comparison work. It is designed for the purpose of quantifying the image intensity change caused by streaks between the rods. The ROIs are shown in Figure 7 . ROI $\mathrm{RI}_{S I}$ are the two rectangles for SI calculation. Each rectangle is $45^{*} 94$ pixels. They are carefully defined so as to include line profiles with two bright and two dark streaks. A total of 90 lines are drawn in $\mathrm{ROI}_{S I}$. An example line profile is plotted in Figure 9.

The following formulas are used:

$$
\begin{aligned}
I_{a} & =\frac{I_{p 1}+I_{p 2}}{2} \\
I_{b} & =\frac{I_{v 1}+I_{v 2}}{2} \\
S I & =\frac{1}{n} \times \sum_{i=1}^{n} \frac{I_{a i}-I_{b i}}{\max \left(I_{a i}, I_{b i}\right)-M_{b}+C}
\end{aligned}
$$

Where $I_{p 1}$ and $I_{p 2}$ are the two image intensity peaks, and $I_{v 1}$ and $I_{v 2}$ are the two valleys. Notice that the pixel indices of $I_{p 1}, I_{p 2}, I_{v 1}$ and $I_{v 2}$ are determined on the uncorrected images. Peak and valley image intensities on corrected images are defined as the image intensities corresponding to these same pixel indices. $M_{b}$ is the average grey value of $\mathrm{ROI}_{b}$. C is defined as per the CNR section. The value of SI is positive in uncorrected images, as $\mathrm{I}_{a}$ is greater than $\mathrm{I}_{b}$. Decreasing SI values are an indication of decreasing severity of the streaks, hence better beam hardening correction.

\subsection{Cupping effect index (CI)}

The CI metric quantifies the image intensity change caused by cupping effects at the boundary of the rod. The ROIs are shown in Figure 7. On the rod in $\mathrm{ROI}_{C I}$, a total of 180 lines are used for CI calculation. Each of them extends $0.25 \mathrm{~mm}$ outside the rod in both directions. An example line profile is plotted in Figure 9. The following formula is used:

$$
C I=\frac{1}{n} \times \sum_{i=1}^{n} \frac{I_{a i}-I_{b i}}{\max \left(I_{a i}, I_{b i}\right)-M_{b}+C}
$$

The notations are in accordance with the previous section. However, as there is only one valley in each line profile, $\mathrm{I}_{b}$ equals $\mathrm{I}_{v}$ here. 

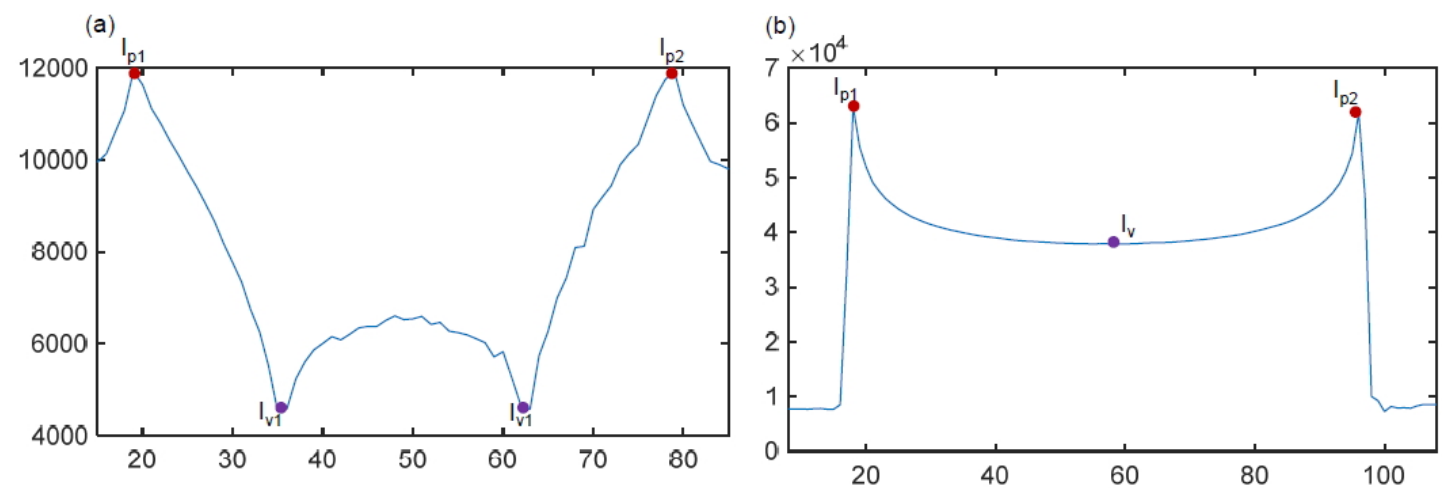

Figure 9: (a) An example SI line profile. The peaks and valleys correspond to the bright and dark streaks that the line traverses. (b) An example CI line profile. The peaks and valley correspond to the edge and centre of the rod that the line traverses.

\subsection{Dimensional measurement}

CT metrology is a recent advance in industry. Its advantage lies in its ability of non-destructive measurement of the internal features. The mechanism of how the BHC algorithms affect the dimensional properties of the phantom needs to be checked, because it determines the reliability of CT as a technique for metrology.

The dimensional offsets from the reference measurements are shown in the results. The offset is calculated by:

Offset $(\mu \mathrm{m})=$ measured radius - reference radius

The corresponding monochromatic data set is used for dimensional measurement reference values. The simulation process inevitably causes errors, thus the real dimension of the phantom never equals to its nominal value. The near-optimal solution is to use the measurement from the monochromatic data set as the reference.

The measurement is performed by fitting a cylindrical surface point cloud onto the rod with VGStudio MAX 2.2.6 (Volume Graphics, Germany). The advanced mode is used for edge detection. It is better at overcoming grey value local variations and hence provides a more stable edge detection in the presence of noise. The search distance is set as $0.2 \mathrm{~mm}$, which is 2 voxels from each side of the detected edge. This distance should be able to cover the local variation, yet 
not elongate the edge detection time too much.

\subsection{General methodology}

The phantom projection simulations are performed using aRTist software (BAM Federal Institute for Materials Research and Testing, Germany) under various energy settings (monochromatic at $58 \mathrm{kV}$, and polychromatic settings at $120 \&$ $220 \mathrm{kVp}$ ). $58 \mathrm{kV}$ was used as it is the effective energy level of the $120 \mathrm{kVp}$ spectrum. Because actual X-ray projections are subject to quantum noise, we simulate all the data sets both noise-free and with quantum noise. Gaussian noise is superimposed on the projections, the standard deviation of which is proportional to the square root of a given projection image intensity (Duvauchelle et al. 2000). For a mean background intensity equals 58,000 , the standard deviation is ca. 400. Scattering is not simulated, as the focus of this study is on beam hardening.

The spectral simulation is conducted with aRTist. Since aRTist suffers interpolation error at the low end $(<40 \mathrm{kV})$ of the spectrum, a $2 \mathrm{~mm}$ Aluminium filter is applied to the source to diminish the low energy part. In order for a BHC algorithm to achieve its full potential, the same spectra are applied to the projection simulation and the algorithm. The spectral mismatch between the image and the algorithm can thus be avoided. Specifications of the aRTist simulation can be found in Table 2. FBP reconstruction is conducted with CTPro (X-Tek Systems Ltd., UK). We then compare the BHC methods with multiple metrics. The phantom dimensions are subsequently measured in VGStudio MAX 2.2.6. The single- \& multi-material linearization and dual-energy methods are implemented in MATLAB R2015a on a laptop computer with 8GB RAM and a $2.5 \mathrm{GHz}$ quad-core processor. It takes about $3 \mathrm{~h}$ for dual-energy to process one data set. Both single- and multi-material linearization methods cost $1.5 \mathrm{~h}$. The iterative method is implemented in IDL on a desktop computer with 32GB RAM and a $3.6 \mathrm{GHz}$ quad-core processor. It takes $6 \mathrm{~h}$ to run on a Tesla C2075 GPU.

\section{Results}

\subsection{Contrast-to-noise ratio (CNR)}

Figure 11(a) shows the results without noise. There are several factors that may affect the CNR value. Table 3 presents these factors separately. $M$ is the mean grey value and $\sigma$ is the standard deviation of the grey value. The absolute percentage change from the corresponding monochromatic value is given inside the 


\begin{tabular}{c|c}
\hline Source type & $\begin{array}{c}\text { Reflection, } \\
\text { tungsten target }\end{array}$ \\
\hline Source filter & 2mm aluminium \\
\hline Voltage range & $\begin{array}{c}\text { Monochromatic: } 58 \mathrm{kV} \\
\text { Polychromatic: } 120 \& 220 \mathrm{kVp}\end{array}$ \\
\hline Focal spot & Point \\
\hline Voxel size & $0.05 \mathrm{~mm}$ \\
\hline Projection number & 3600 \\
\hline Detector & $\begin{array}{c}0.2 \mathrm{~mm} \text { pixel size, } \\
2000 \times 2000 \text { pixels, } \\
16 \text { bit dynamic range, } \\
\text { energy-summing flat panel }\end{array}$ \\
\hline
\end{tabular}

Table 2: Specifications of the aRTist simulation.

parenthesis. The mean and standard deviation of the background image intensity hardly change across data sets. Therefore, they have no influence on CNR. The change is mainly caused by the mean and standard deviation of the rod. The monochromatic simulation yields the same CNR values for both $\mathrm{ROI}_{1}$ and $\mathrm{ROI}_{2}$. In the polychromatic data set, the value of $\mathrm{CNR}_{1}$ is greater than that of $\mathrm{CNR}_{2}$, because $\mathrm{ROI}_{2}$ is affected drastically by streaks whereas $\mathrm{ROI}_{1}$ is not. 


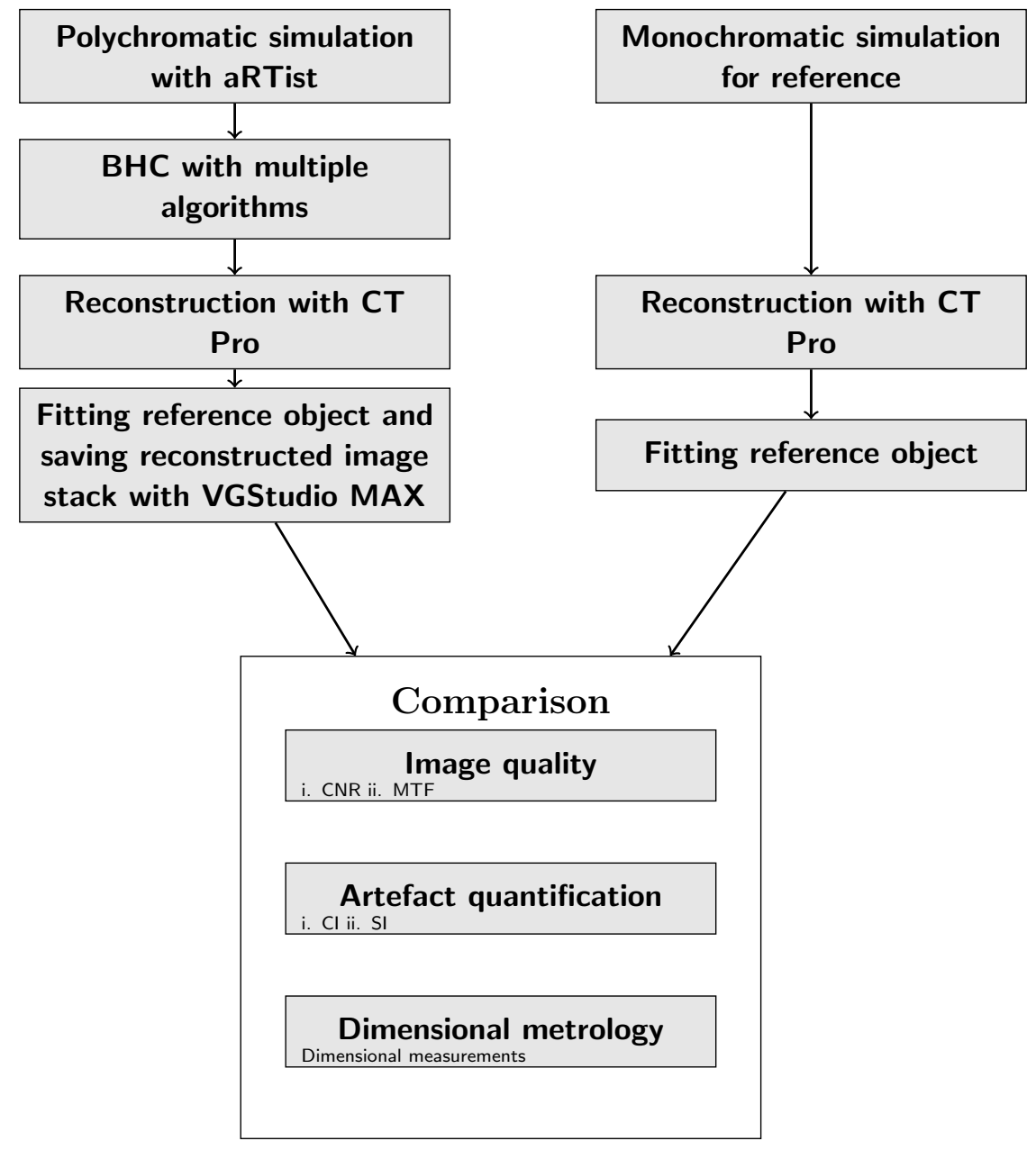

Figure 10: Flow chart of the experimental plan. 


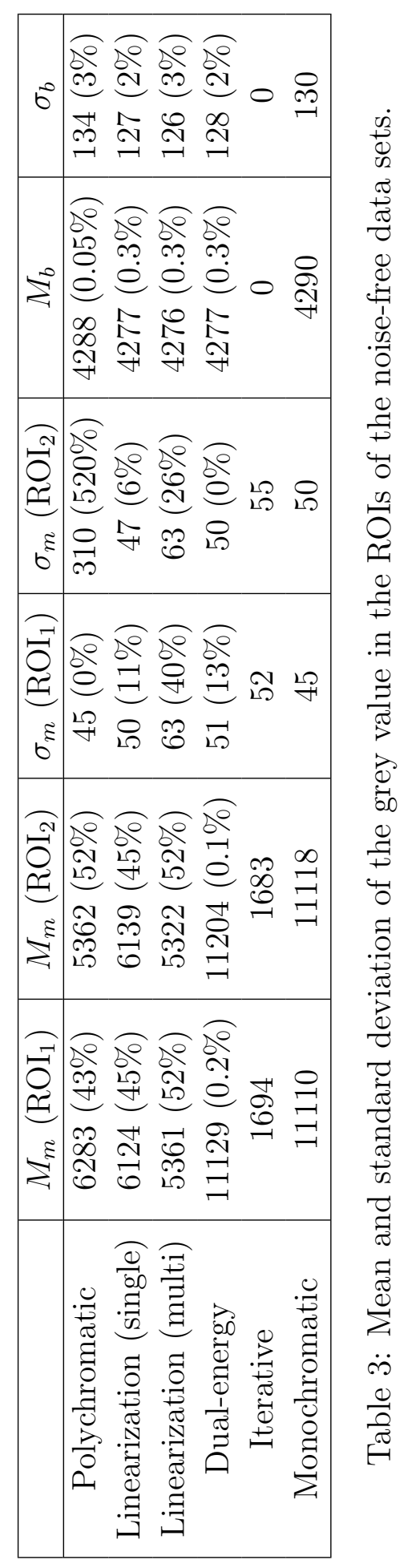


The dual-energy algorithm brings the $\mathrm{CNR}$ values of $\mathrm{ROI}_{1}$ and $\mathrm{ROI}_{2}$ back to the monochromatic values. The linearization algorithms also bring the values of $\mathrm{ROI}_{1}$ and $\mathrm{ROI}_{2}$ in accordance with each other, but both of them are lower than the values of the monochromatic data set. The iterative method yields a CNR value at ca. $5 E-4$. It is lower than the dual-energy value (ca. $7.5 E-4$ ), but higher than the linearization values (ca. $1.3 E-4)$. In the linearization data sets, the CNR value is $17 \%$ of the reference. Although the value is low, the plastic rod is legible.

The results of the data sets with quantum noise are plotted in Figure 11(b). Noise increases the standard deviation of both the rod and background, so the CNR methods of most data sets are lower. The only exception is the data set of the iterative method. It has integrated a noise model, so noise can be restrained in reconstruction.

(a)

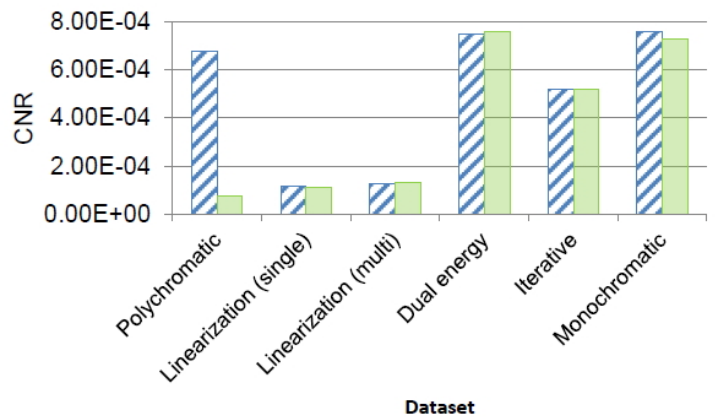

(b)

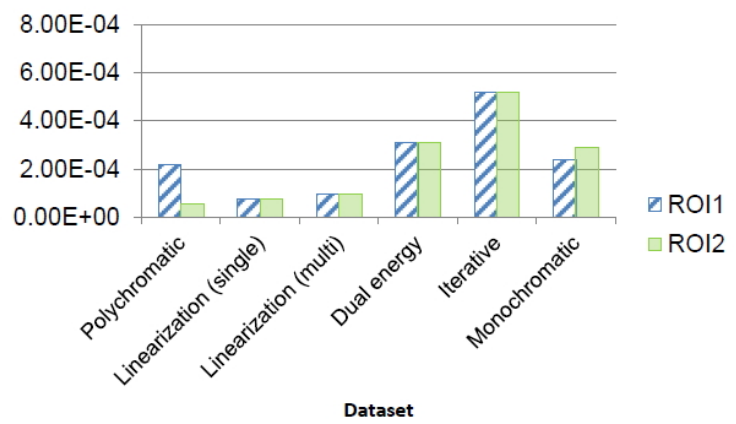

Figure 11: CNR values for the simulations before (polychromatic) and after BHC in comparison with the CNR values of a monochromatic simulation. (a) No quantum noise, (b) with quantum noise. 


\subsection{Modulation transfer function (MTF)}

Figure 12 compares the results from all methods investigated. The monochromatic simulation yields a value of 12.4 , whereas the polychromatic simulation yields 12.2. They are similar because the rod in $\mathrm{ROI}_{c}$ is within a PPS rod that is only weakly affected by beam hardening induced cupping effect. All algorithms yield MTF values close to the monochromatic values, but the iterative method value stands out with a value of 18 .

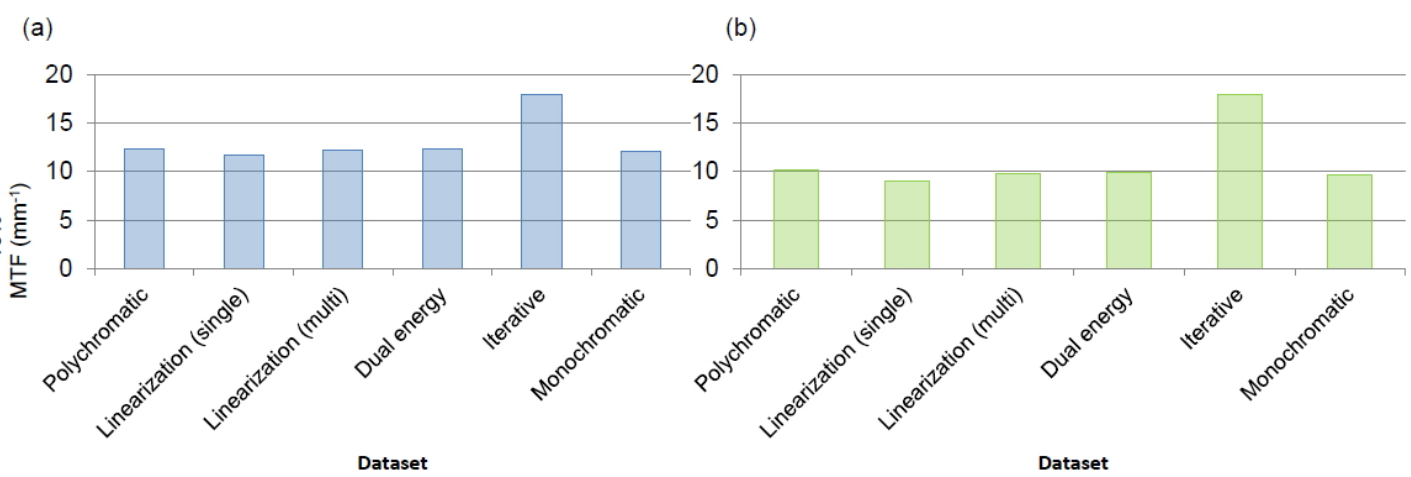

Figure 12: $10 \% \mathrm{MTF}$ values for the simulations before (polychromatic) and after BHC in comparison with the $10 \% \mathrm{MTF}$ of a monochromatic simulation. (a) No quantum noise, (b) with quantum noise.

The edge spread function (ESF) plots of the iterative and the monochromatic data are shown in Figure 9. Compared to the smooth curve of the monochromatic ESF, a sharp peak at a radius of $145 \mu \mathrm{m}$ can be observed in the iterative one, which indicates a sudden image intensity rise near the boundary of the rod in $\mathrm{ROI}_{c}$. It gives an aliasing pattern which appears to be similar to cupping effects. Since the iterative method reduced beam hardening streaks, it is likely that the cupping effects are also reduced. Therefore, the main reason for the abnormal $10 \% \mathrm{MTF}$ is the additional aliasing pattern.

\subsection{Streak index (SI)}

Figure 14 depicts the central slices of the reconstructed images. The single-material linearization method clearly reduced the streaks between the two adjacent metal rods, but streaks are induced in the vicinity of the PPS rods. Interestingly, the appearance of the streaks has been changed comparing to the polychromatic 
(a)

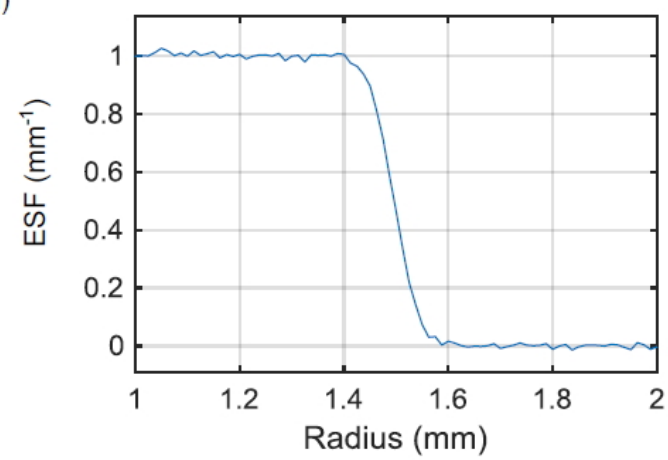

(b)

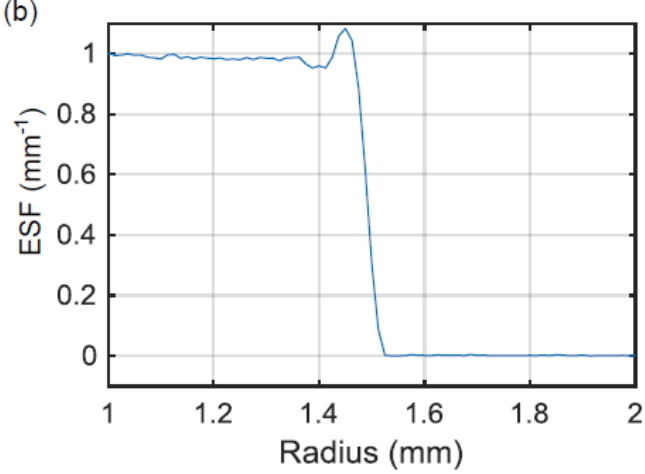

Figure 13: ESFs (without noise). (a) Monochromatic, (b) iterative.

image. The multi-material linearization, dual-energy, and the iterative methods can eliminate the visual streaks on the images.

SI values are depicted in Figure 15(a). The monochromatic simulation yields a value of 0 , whereas the uncorrected data yields a value of 0.11 . The iterative method yields an SI value equal to 0, meaning that it eliminated all the streaks in the background. The linearization and dual-energy methods yield values ca. $1 E-3$. The remaining artefacts are mostly caused by the limited number of ray paths being calculated by aRTist. As X-ray is continuous, roundoff errors in the discretization process of simulation is inevitable. It is worth mentioning that since single-material linearization was applied to Ti, it works equally well as other methods in the defined ROI.

The results of the algorithm comparison on the noisy data sets are shown in Figure 15(b). The addition of noise has not affected the performance of any of the algorithms in correcting the streaks.

\subsection{Cupping effect index (CI)}

Figure 16 plots line profiles of all data sets. Substantial cupping effects are present in the polychromatic line profile, indicating a severe image intensity drop from the surface to the centre of the rod. On the contrary, a flat surface can be observed in the monochromatic line profile, which indicates that the image intensities within the rod are uniform. The image intensities of the multi-material linearization data set are less uniform than in the monochromatic data set. The cupping effect looks prominent in the iterative line profile.

The results of the algorithm comparison on the noise-free data sets are shown 


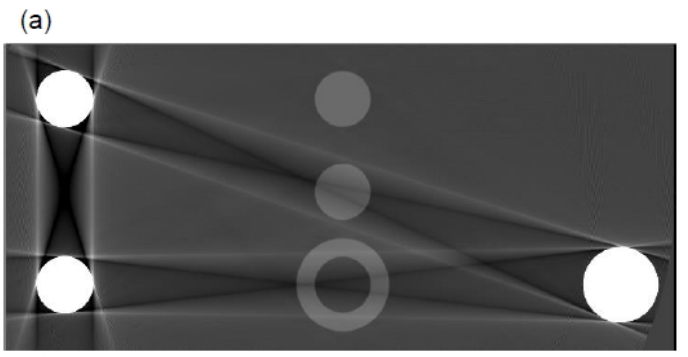

(b)

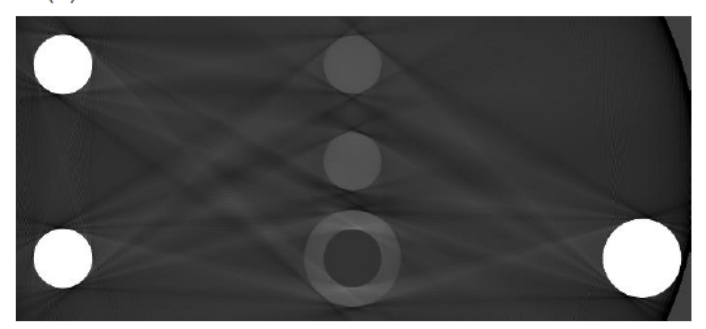

(d)

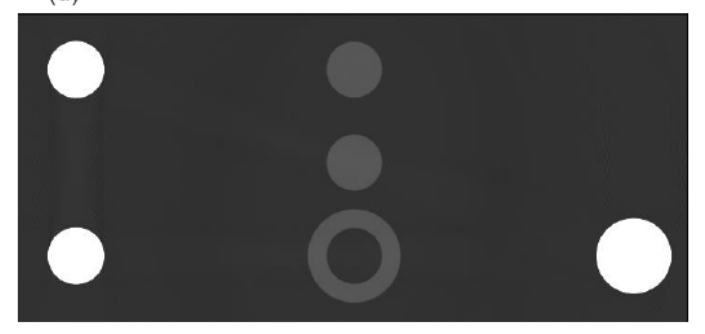

(c)

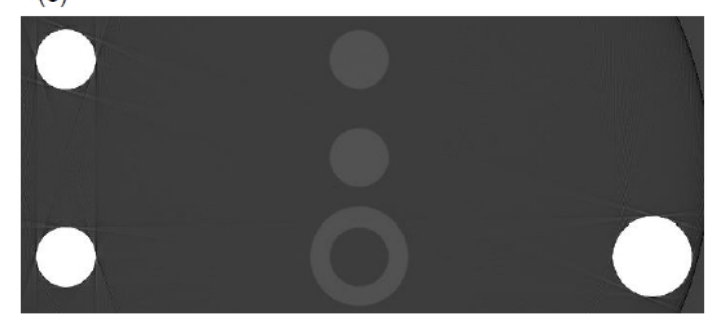

(e)

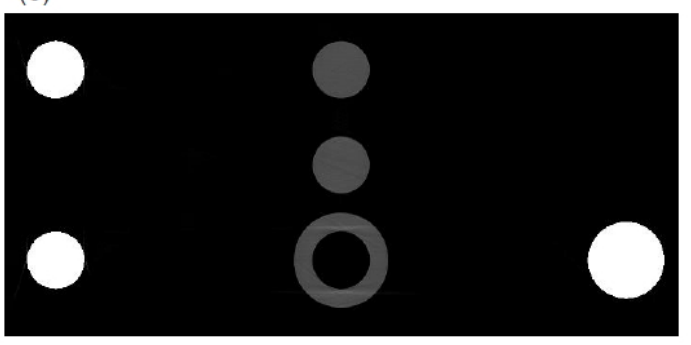

Figure 14: Reconstructed central slice (without noise) of the data sets. (a) Polychromatic, (b) single-material linearization, (c) multi-material linearization, (d) dual-energy, (e) iterative.

in Figure 17(a). The monochromatic simulation yields a value of 0 as expected, and the uncorrected data yields a value of 0.15 . The multi-material linearization method yields a value of 0.009 , whereas the dual-energy method yields -0.003 . Generally speaking, both methods can efficiently reduce cupping effects, but dualenergy introduces a slight over-correction.

The iterative method yields a value of 0.07 , which seems indicating only a partial correction. However, the insights from the MTF section can indicate that the overshoot of image intensity at the boundary is caused by aliasing introduced by the algorithm instead of the original cupping effects.

The results of the algorithm comparison on the noisy data sets are presented in Figure 17(b). It is evident that the addition of noise has not affected the 
(a)

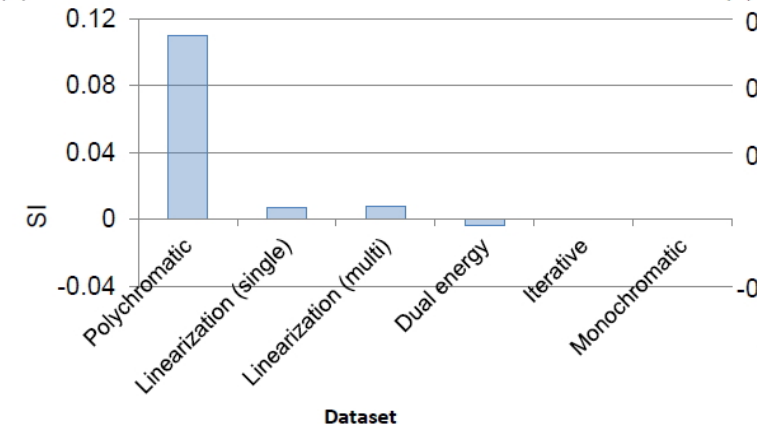

(b)

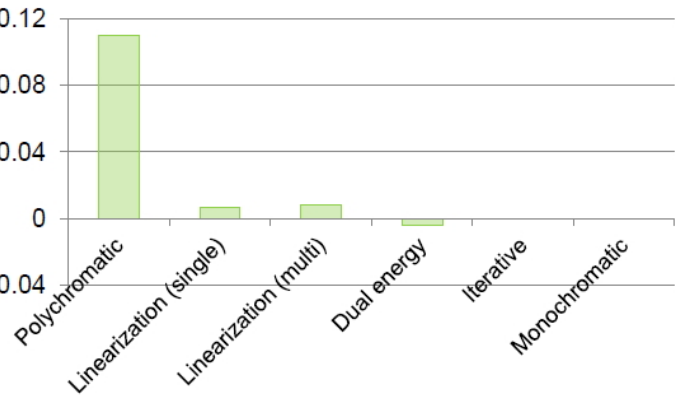

Figure 15: SI values for the simulations before (polychromatic) and after BHC in comparison with the SI of a monochromatic simulation. (a) No quantum noise, (b) with quantum noise.

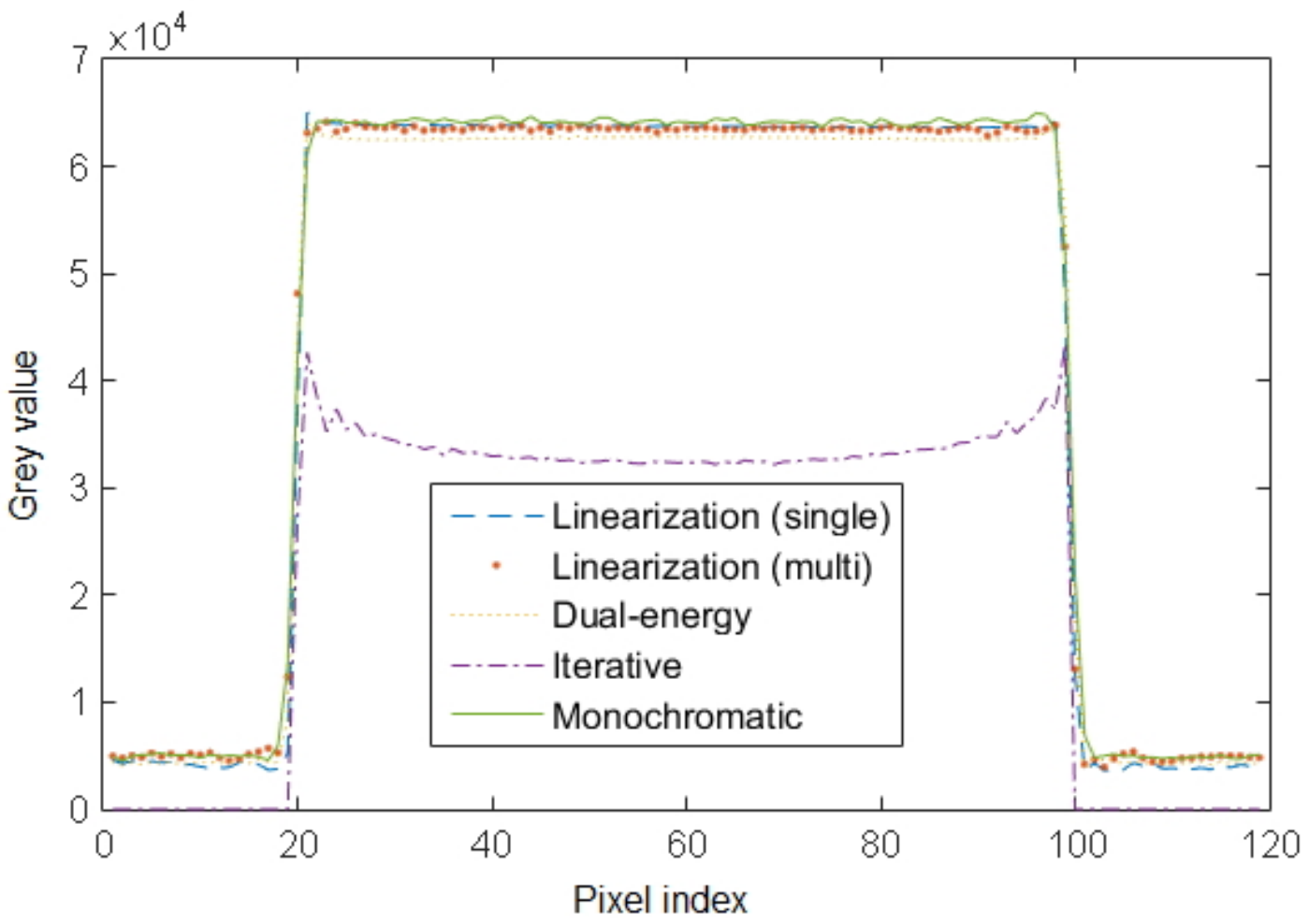

Figure 16: Line profiles (without noise) along the centre of a Ti rod.

performance of any of the algorithms in correcting the cupping effects. 
(a)

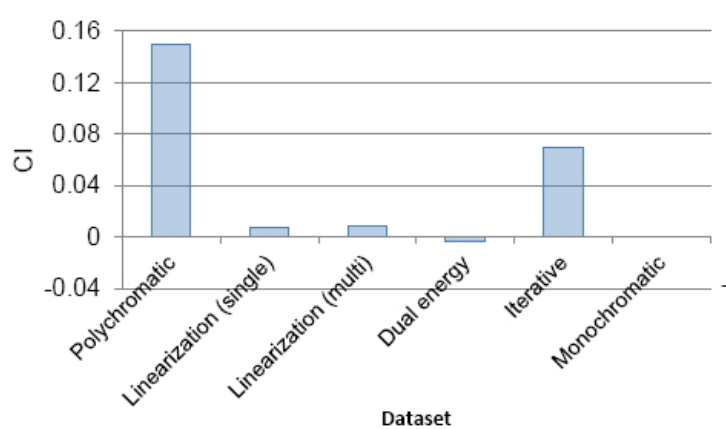

(b)

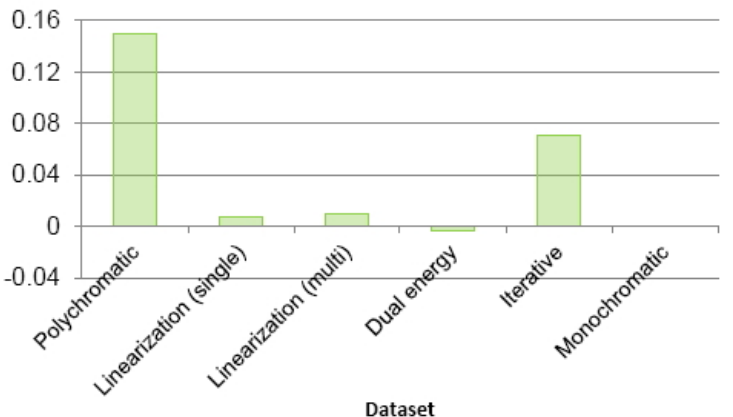

Figure 17: CI values for the simulations before (polychromatic) and after BHC in comparison with the CI of a monochromatic simulation. (a) No quantum noise, (b) with quantum noise.

\subsection{Dimensional measurement}

Figure 18 and Table 4 show the dimensional offsets. Because the iterative method leads to a substantially larger dimensional offset than the other methods due to the edge idiosyncrasy, its result is not included in the figure. Both in the noisefree and in the noisy uncorrected data sets, the offsets are larger for the Ti rods than for PPS ones. Rods of the same material yield similar offsets. The averaged absolute offsets of each material are presented in Table 4. The percentage change from the corresponding polychromatic value is given in the parenthesis.

\begin{tabular}{|c|c|c|c|c|}
\hline & \multicolumn{2}{|c|}{ No noise } & \multicolumn{2}{c|}{ With quantum noise } \\
\hline & $\operatorname{Ti}(\mu \mathrm{m})$ & $\operatorname{PPS}(\mu \mathrm{m})$ & $\operatorname{Ti}(\mu \mathrm{m})$ & PPS $(\mu \mathrm{m})$ \\
\hline Linearization (single) & $2.65(-71 \%)$ & $5.10(963 \%)$ & $2.93(-68 \%)$ & $4.93(927 \%)$ \\
\hline Linearization (multi) & $1.89(-80 \%)$ & $0.26(-42 \%)$ & $3.23(65 \%)$ & $0.40(-17 \%)$ \\
\hline Dual-energy & $0.27(-97 \%)$ & $0.25(-42 \%)$ & $3.70(60 \%)$ & $0.66(37.5 \%)$ \\
\hline Iterative & $25.20(172 \%)$ & $10.68(2125 \%)$ & $0.27(-97 \%)$ & $9.93(1969 \%)$ \\
\hline Polychromatic & 9.28 & 0.48 & & \\
\hline
\end{tabular}

Table 4: The averaged absolute dimensional offsets by the material.

In the noise-free result, offsets are reduced prominently in both the dualenergy and the multi-material linearization data set. The iterative method did not improve the dimensional accuracy. The single-material linearization method reduced the offsets of the Ti rods, but it failed to reduce offsets of the PPS rods. 
(a)

(b)

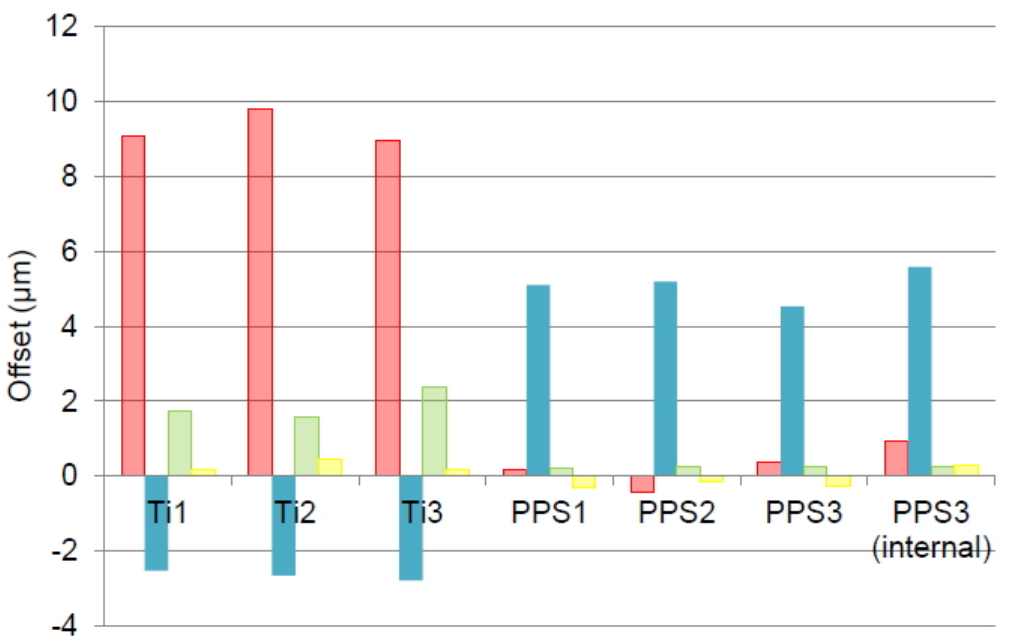

Dual energy

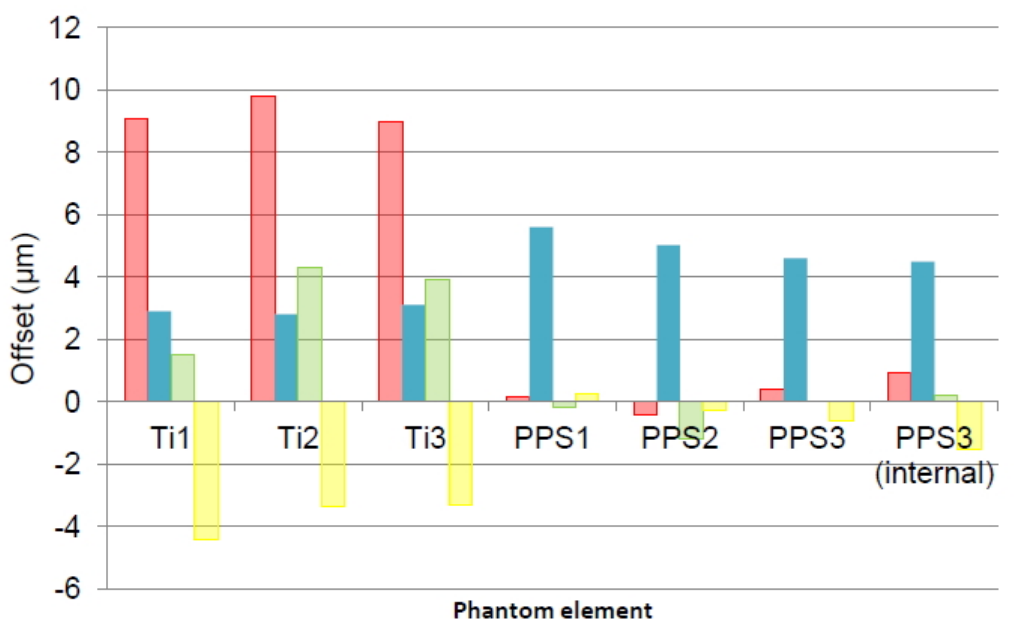

$\square$ Polychromatic

- Linearization (single)

$\square$ Linearization (multi)

Dual-energy

Figure 18: Dimensional offsets. (a) No quantum noise, (b) with quantum noise.

When noise presents, all the methods reduced the offsets of the Ti rods, but increased the offsets of the PPS rods. This is because the low contrast material edge detection is more sensitive to noise.

\section{Discussion}

In this work, the performance of four BHC algorithms was compared. The dualenergy method outperformed other algorithms in the contrast resolution, the artefact quantification and dimensional measurement accuracy. The drawbacks of the dual-energy approach are its dual-data set requirement and expensive 
computational cost.

The single-material linearization method yielded partial correction. The cupping effects on the Ti rods and the streaks between them were eliminated, but the streaks in the vicinity of the PPS rods remained. This subsequently affected the dimensional measurement accuracy of the PPS rods. The multimaterial linearization method was preferred over the single-material linearization because it was able to linearize both materials. One drawback of linearization is it decreases the low contrast detectability of the image.

The cause of the decreased contrast resolution is the underestimation of the low contrast material contribution. In the single-material linearization, as the polynomial coefficients are calculated to correct $\mathrm{Ti}$, it yields lower logarithmic transmission value of PPS. For the multi-material linearization, although each material is linearized separately, an underestimation of the low contrast material contribution happens when pixels belong to Ti are not totally segmented out from PPS.

The statistical iterative algorithm 'IMPACT' has a different nature compared to FBP based methods. The iterative approach seeks a solution to fit every measured data set, while the FBP approach always assumes that the data are exact, consistent and complete. Therefore, the statistical iterative algorithm has superior performance in the presence of noise. Another claimed advantage of this algorithm is the robustness with sparse projections, which we did not explore. IMPACT eliminated all the streaks. However, it did not work well in reducing the cupping effects on the Ti rods, which is liable to be caused by the aliasing patterns introduced by the algorithm rather than by the original cupping effects. It failed to improve the dimensional measurement accuracy for the same reason. Its iterative nature requires a compromise between the computational capacity and performance for big data sets.

The cause of the overshoot artefact of the iterative method remains a tough question to be discussed. An explanation is given by Zbijewski and Beekman (Zbijewski \& Beekman 2003). The iterative method assumes the reconstructed image is voxelized (or discretized), whereas FBP assumes it is continuous. The iterative methods often attempt to compensate for the inappropriate voxelization by introducing high-valued voxels on the sharp edges (Zbijewski \& Beekman 2006). The overshoot artefact could be reduced by conducting the reconstruction on a finer grid, but this requires a higher computational load. Another possible approach is to apply filtering to smooth out the image. It is not based on physical principles and the choice of the filter function is somewhat arbitrary. 


\section{Acknowledgements}

This work has received funding from the European Union's Seventh Framework Programme under grant agreement No. 607817. We would like to express our thanks to Dr Heather Durko of Nikon Research Corporation of America for providing the implementation of the Alvarez algorithm. 


\section{References}

Alvarez, R. E. \& Macovski, A. (1976), 'Energy-selective reconstructions in x-ray computerised tomography', Physics in medicine and biology 21(5), 733.

Brabant, L., Pauwels, E., Dierick, M., Van Loo, D., Boone, M. \& Van Hoorebeke, L. (2012), 'A novel beam hardening correction method requiring no prior knowledge, incorporated in an iterative reconstruction algorithm', $N d t$ \& $E$ International 51, 68-73.

Brooks, R. A. \& Di Chiro, G. (1976), 'Beam hardening in X-ray reconstructive tomography', Physics in medicine and biology 21(3), 390.

Bushberg, J. T. \& Boone, J. M. (2011), The essential physics of medical imaging, Lippincott Williams \& Wilkins.

De Man, B., Nuyts, J., Dupont, P., Marchal, G. \& Suetens, P. (2001), 'An iterative maximum-likelihood polychromatic algorithm for ct', Medical Imaging, IEEE Transactions on 20(10), 999-1008.

Duvauchelle, P., Freud, N., Kaftandjian, V. \& Babot, D. (2000), 'A computer code to simulate X-ray imaging techniques', Nuclear Instruments and Methods in Physics Research Section B: Beam Interactions with Materials and Atoms 170(1), 245-258.

Elbakri, I. A. \& Fessler, J. A. (2002), 'Statistical image reconstruction for polyenergetic X-ray computed tomography', Medical Imaging, IEEE Transactions on $\mathbf{2 1}(2)$, 89-99.

Friedman, S. N., Fung, G. S., Siewerdsen, J. H. \& Tsui, B. M. (2013), 'A simple approach to measure computed tomography (ct) modulation transfer function (mtf) and noise-power spectrum (nps) using the american college of radiology (acr) accreditation phantom', Medical physics 40(5), 051907.

Hammersberg, P. \& Mångård, M. (1998), 'Correction for beam hardening artefacts in computerised tomography', Journal of X-ray Science and Technology 8(1), 7593.

Herman, G. T. (1979), 'Correction for beam hardening in computed tomography', Physics in Medicine and Biology 24(1), 81.

Herman, G. T. (2009), Fundamentals of computerized tomography: image reconstruction from projections, Springer.

Herman, G. T. \& Trivedi, S. S. (1983), 'A comparative study of two postreconstruction beam hardening correction methods', Medical Imaging, IEEE Transactions on $\mathbf{2}(3), 128-135$. 
Joseph, P. M. \& Ruth, C. (1997), 'A method for simultaneous correction of spectrum hardening artifacts in ct images containing both bone and iodine', Medical Physics 24(10), 1629-1634.

Joseph, P. M. \& Spital, R. D. (1978), 'A method for correcting bone induced artifacts in computed tomography scanners.', Journal of computer assisted tomography 2(1), 100-108.

Kalender, W. A., Perman, W., Vetter, J. \& Klotz, E. (1986), 'Evaluation of a prototype dual-energy computed tomographic apparatus. i. phantom studies', Medical physics 13(3), 334-339.

Lehmann, L., Alvarez, R., Macovski, A., Brody, W., Pelc, N., Riederer, S. \& Hall, A. (1981), 'Generalized image combinations in dual KVP digital radiography', Medical physics 8(5), 659-667.

Liu, X., Yu, L., Primak, A. N. \& McCollough, C. H. (2009), 'Quantitative imaging of element composition and mass fraction using dual-energy CT: three-material decomposition', Medical physics 36(5), 1602-1609.

McDavid, W. D., Waggener, R. G., Payne, W. H. \& Dennis, M. J. (1977), 'Correction for spectral artifacts in cross-sectional reconstruction from X-rays', Medical physics 4(1), 54-57.

Nalcioglu, O. \& Lou, R. (1979), 'Post-reconstruction method for beam hardening in computerised tomography', Physics in medicine and biology 24(2), 330.

Van de Casteele, E., Van Dyck, D., Sijbers, J. \& Raman, E. (2004), 'A model-based correction method for beam hardening artefacts in x-ray microtomography', Journal of X-ray Science and Technology 12(1), 43-57.

Van Slambrouck, K. \& Nuyts, J. (2012), 'Metal artifact reduction in computed tomography using local models in an image block-iterative scheme', Medical physics 39(11), 7080-7093.

Yan, C. H., Whalen, R. T., Beaupre, G. S., Yen, S. Y. \& Napel, S. (2000), 'Reconstruction algorithm for polychromatic CT imaging: application to beam hardening correction', Medical Imaging, IEEE Transactions on 19(1), 1-11.

Zbijewski, W. \& Beekman, F. J. (2003), 'Characterization and suppression of edge and aliasing artefacts in iterative x-ray ct reconstruction', Physics in Medicine Es Biology 49(1), 145.

Zbijewski, W. \& Beekman, F. J. (2006), 'Comparison of methods for suppressing edge and aliasing artefacts in iterative X-ray CT reconstruction', Physics in Medicine $\mathscr{G}$ Biology 51(7), 1877. 